\title{
Delirium and it's Risk Factors among Elderly Patients
}

\author{
Sasikala R. Umesh ${ }^{1}$, Selva Titus Chacko ${ }^{2}$, Amalorpavamari Lucas ${ }^{3}$, Aruna Sundararaj ${ }^{4}$ \\ ${ }^{1} \mathrm{MSc}(\mathrm{N})$ Reader \\ ${ }^{2} \mathrm{MSc}(\mathrm{N}) ., \mathrm{PhD}(\mathrm{N})$ Principal \& Professor \\ ${ }^{3} \mathrm{MSc}(\mathrm{N})$ Professor, Medical Surgical Nursing \\ ${ }^{4} \mathrm{BSc}(\mathrm{N})$ Charge Nurse, College of Nursing, CMC, Vellore
}

\begin{abstract}
Delirium is a common syndrome prevalent among hospitalized elderly patients. The prevalence is estimated to be 11 to 42 percent among elderly patients admitted in medical wards. The consequences of delirium could be significant such as an increase in mortality in the hospital, long-term cognitive decline, loss of autonomy and increased risk for long term hospitalization. Despite being a common condition, delirium remains under-recognized, poorly understood and inadequately managed. Therefore a descriptive study was conducted in private medical wards of a Tertiary care hospital in South India, to assess the prevalence of delirium and it's risk factors among hospitalized elderly patients. 150 participants were included in the study by total enumerative sampling technique. The short version of Confusion Assessment Method (CAM) and Delirium Risk Assessment Tool (DRAT) were used to assess prevalence and its risk factors. $74 \%$ of the participants had risk factors for delirium of which $11 \%$ of them had delirium. There was statistically significant association between delirium and its risk factors. Findings of the study helped to develop a protocol on multicomponent nonpharmacological nursing interventions, which will enable nurses to identify delirium and to care for elderly patients with delirium.
\end{abstract}

Keywords: Delirium, Delirium risk factors, Elderly, Delirium protocol

\section{Introduction and Need for the Study}

Delirium is a disturbance of consciousness with impaired attention and disorganized thinking that develops rapidly with incidence of underlying physiological or medical condition (American Psychiatric Association, 2000). Delirium among elderly patients with medical and surgical disease conditions is $10 \%$ to $15 \%$ on admission and inhospital occurrence is $10 \%$ to $40 \%$ (Fann,J.R 2000). Studies indicate that delirium is unrecognized in $66 \%$ to $84 \%$ of hospitalized elderly patients (Pisani et al., 2006). Advanced age and dementia are the most important risk factors for delirium. Pain, dehydration, infections, stroke, metabolic disturbances, and surgery are the most common risk factors. The risk of delirium increases with the number of risk factors.

Identification of risk factors by prompt and appropriate assessment with Multifactorial Model for Delirium and continued surveillance are the corner stones of delirium prevention (Inouye,S.K.Van,C.H.,Alessi,C.A.,et al.,1990). Nurses are in the best position to assess the risk factors and detect delirium in an early stage. Early identification and prompt management influences the outcome of medical treatment. Due to advancement of medicine and increased awareness among elderly adults the geriatric hospitalized patients have increased significantly. From the morbidity point of view, almost $50 \%$ of the Indian elderly have chronic diseases and five per cent suffer from immobility. The elderly population is the most vulnerable group for delirium. This scenario demands the nurses to be competent in caring for elderly patients with possible delirium. Hence, this study was conducted to assess the prevalence of delirium and to identify it's risk factors among hospitalized patients in tertiary care hospital.

\subsection{Objectives}

1) To assess the prevalence of delirium and it's risk factors among elderly patients

2) To determine the association between delirium and selected demographic and clinical variables

3) To determine the association between delirium and it's risk factors

\section{Methodology}

Descriptive design was utilized. The study was conducted in private medical wards in multispecialty Tertiary care Training and Research hospital in South India. Population consisted of elderly patients $>60$ years of age, who are admitted with medical illness. 150 participants were chosen using total enumerative sampling technique.

\section{Instruments and scoring}

The instrument includes three sections:

Section 1: Demographic characteristics: age, gender, mode of payment, previous history of delirium and co-morbidities (Diabetes Mellitus, Hypertension, Cardiovascular Diseases, Renal Disorders, Infection, others).

Section II: Short version of Confusion Assessment Method (CAM) tool was used to assess the prevalence of delirium. It has 4 features: A- Acute onset and Fluctuating course, BInattention, C- Disorganized thinking and D- Altered level of consciousness. The diagnosis of delirium requires presence of both $\mathrm{A}$ and $\mathrm{B}$ and presence of either $\mathrm{C}$ or $\mathrm{D}$. 


\section{International Journal of Science and Research (IJSR) \\ ISSN (Online): 2319-7064}

Index Copernicus Value (2016): 79.57 | Impact Factor (2015): 6.391

CAM is a standardized tool with a sensitivity of $94-100 \%$, specificity of $90-95 \%$ and inter-observer reliability of kappa $=0.81-1.0$ (Inouye, 1990).

Section III: Delirium Risk Assessment Tool (DRAT) is a standardized tool. It has two components. Component I assesses the age. Component II consists of 4 features, which assesses features like visual impairment, severe illness, cognitive impairment and dehydration. Risk of delirium is confirmed when the subject has component I and has at least one of the risk factors in component II. The confidence rating of DRAT tool has median score from 3 to 4 (range 25, p > 0.00), Shah, N., Knifton,C., Dowsing, T (2013).

\section{Data Collection}

The study was conducted with the permission from the Research Committee of the Institution. Written consent was obtained from all the study participants. Confidentiality and anonymity was maintained. The investigator assessed the presence of delirium and risk factors.

\section{Data Analysis}

SPSS 18.0 was used to perform all statistical analysis. Descriptive statistics like frequency and percentage were used to analyze the prevalence of delirium and it's risk factors. Quantitative variables were summarized using mean and standard deviation. Chi-square was used to find the association between delirium, demographic variables and risk factors. $\mathrm{P}$ value $<0.05$ was considered as statistically significant.

\section{Results and Discussion}

Analysis of demographic data revealed that majority of the participants $(48 \%)$ were between the age group of 60 69years. $54.7 \%$ of the participants were males. $92.7 \%$ of the participants had no previous history of delirium. $49 \%$ of the participants had 3-4 co morbidities. Similar findings were also reported by Varghese,N.C (2012).

Among the study participants $11 \%$ had delirium. This finding is similar to a point prevalence study done by Riyan ,D.J.,et al.,(2017), which revealed that $17.6 \%$ had delirium. Whereas, study conducted by Varghese, N.C (2012) in general medical wards in a tertiary hospital showed that 44 $\%$ of the older adults had delirium. Lower rate of delirium prevalence in the present study could be due to the high education, knowledge of the health issues and access to the health care on time.

The study findings revealed that $74 \%$ of the participants had risk factors for delirium. These findings are well supported by a study done by Eduard. E, et al,2013, which states that infections, such as a urinary tract infection or pneumonia, are probably some of the most common precipitants of delirium and may be present in 34-64\% of the hospitalized patients with delirium. Multi factorial Model for Delirium suggests that the risk of delirium increases with the number of risk factors present (Inouye,S.K., Van,C.H. Alessi,C.A.,et al (1990). This study findings signify the importance of identification of high-risk patients, risk factors, prompt and appropriate treatment and continued surveillance for delirium prevention.

A statistically significant association was found in age, male gender and previous history of delirium as shown in Table-1. These findings are consistent with the findings of a systematic review and meta-analysis of four studies done by Ahmed S.,Leurent, B, Sampson,E.L (2014), which revealed that old age was significantly associated with increased delirium with a mean difference of 2.74(95\% -CI 0.11,5,38, $\mathrm{P}=0.04)$. Content data bases were screened and reported by Elie.M., et al in 1998, that the five most common risk factors for delirium were dementia, medication, medical illness age, and gender, which is consistent with the present study findings.

Table 1: Association between delirium and demographic and clinical variables $(n=150)$

\begin{tabular}{|c|c|c|c|c|c|c|c|c|c|}
\hline \multirow[t]{2}{*}{ S,No } & \multirow[t]{2}{*}{ Variables } & \multicolumn{2}{|c|}{$\begin{array}{c}\text { Delirium } \\
\text { Present }\end{array}$} & \multicolumn{2}{|c|}{$\begin{array}{c}\text { Delirium } \\
\text { Absent }\end{array}$} & \multicolumn{2}{|c|}{ Total } & \multirow[t]{2}{*}{$\chi^{2}$} & \multirow[t]{2}{*}{$\begin{array}{c}\mathrm{P} \\
\text { Value }\end{array}$} \\
\hline & & No & $\%$ & No & $\%$ & No & $\%$ & & \\
\hline \multirow[t]{4}{*}{1} & Age & & & & & & & & \\
\hline & 60-69yrs & 3 & 4.17 & 69 & 95.83 & 72 & 48.00 & & \\
\hline & 70-79yrs & 7 & 13 & 47 & 87 & 54 & 36.00 & 8.666 & 0.013* \\
\hline & $>\underline{80 y r s}$ & 6 & 25 & 18 & 75 & 24 & 16.00 & & \\
\hline 2 & Gender & & & & & & & & \\
\hline \multirow[t]{2}{*}{3} & Male & 9 & 11 & 73 & 89 & 82 & 54.67 & 8.589 & 0.014* \\
\hline & Female & 7 & 10.30 & 61 & 89.70 & 68 & 45.33 & & \\
\hline \multirow[t]{3}{*}{4} & $\begin{array}{l}\text { Previous history } \\
\text { of delirium }\end{array}$ & & & & & & & & \\
\hline & Yes & 5 & 45.45 & 6 & 54.55 & 11 & 7.33 & 15.076 & $0.000 *$ \\
\hline & No & 11 & 7.92 & 128 & 92.08 & 139 & 92.67 & & \\
\hline
\end{tabular}

The present study revealed that there was statistical significant association between delirium and age, sodium and potassium dyselectrolytemia, hypoxia, mental illness, cognitive impairment and dehydration as presented in Table 2. $37.5 \%$ of the participants with hyponatremia and $33.3 \%$ of the participants with hypokalemia had delirium. These findings are supported by the study conducted by Zieschang T, 2009 who reported that, patients with hyponatremia suffered more often from delirium. A study by Yong TY.,
Huang JE,Lau SY, Li JY(2011), also supports that eleven patients were admitted with severe hyponatremia and ten patients with hypokalemia, of which delirium was present among six patients. This study findings also suggest that $36.4 \%$ had hypoxia, $37.5 \%$ had mental illness, $42.1 \%$ had cognitive impairment and $22.7 \%$ of the participants had dehydration. These findings are consistent with a prospective cohort study by Korevaar. J.C.,(2005), which revealed that cognitive impairment and an increased urea

\section{Volume 6 Issue 12, December 2017}




\section{International Journal of Science and Research (IJSR) \\ ISSN (Online): 2319-7064}

Index Copernicus Value (2016): 79.57 | Impact Factor (2015): 6.391

nitrogen level were the strongest risk factors which increased the risk of delirium by nine fold. The study finding revealed that, none of the study participants had the risk factor of alcohol intoxication.

Table 2: Association between delirium and risk factors $(\mathrm{n}=150)$

\begin{tabular}{|c|c|c|c|c|c|c|c|c|}
\hline \multirow[b]{2}{*}{ Description } & \multicolumn{2}{|c|}{ Delirium Present } & \multicolumn{2}{|c|}{ Delirium Absent } & \multicolumn{2}{|c|}{ Total } & \multirow{2}{*}{$\chi^{2}$} & \multirow[t]{2}{*}{ P Value } \\
\hline & $\mathrm{N}$ & $(\%)$ & $\mathrm{N}$ & $(\%)$ & No & $\%$ & & \\
\hline Age $\geq 60 \mathrm{yrs}$ & 16 & 10.66 & 134 & 89.33 & 150 & 100 & 8.666 & 0.013* \\
\hline \multicolumn{9}{|c|}{ Diabetic Ketoacidosis } \\
\hline Present & 1 & 100.00 & 0 & 0.00 & 1 & 0.67 & & \\
\hline Absent & 15 & 10.07 & 134 & 89.33 & 149 & 99.33 & 8.431 & 0.004* \\
\hline \multicolumn{9}{|l|}{ Dyselectrolytemia } \\
\hline \multicolumn{9}{|l|}{ Sodium } \\
\hline Present & 9 & 37.50 & 15 & 62.50 & 24 & 16.00 & 21.589 & $0.000 *$ \\
\hline Absent & 7 & 5.56 & 119 & 94.44 & 126 & 84.00 & & \\
\hline \multicolumn{9}{|l|}{ Potassium } \\
\hline Present & 5 & 33.33 & 10 & 66.67 & 15 & 10.00 & 8.986 & 0.003* \\
\hline Absent & 11 & 8.14 & 124 & 91.86 & 135 & 90.00 & & \\
\hline \multicolumn{9}{|c|}{ Hypoxia/ Hypercapnia } \\
\hline Present & 4 & 36.4 & 7 & 63.6 & 11 & 7.33 & 8.226 & 0.004* \\
\hline Absent & 12 & 8.6 & 127 & 91.4 & 139 & 92.67 & & \\
\hline \multicolumn{9}{|c|}{ Mental illne or depression } \\
\hline Present & 3 & 37.50 & 5 & 62.50 & 8 & 5.33 & 6.386 & 0.012* \\
\hline Absent & 13 & 9.15 & 129 & 90.85 & 142 & 94.67 & & \\
\hline \multicolumn{9}{|c|}{ Cognitive impairment } \\
\hline Present & 8 & 42.1 & 11 & 57.9 & 19 & 12.67 & 22.566 & $0.000 *$ \\
\hline Absent & 8 & 6.1 & 123 & 93.9 & 131 & 87.33 & & \\
\hline \multicolumn{9}{|l|}{ Dehydration } \\
\hline Present & 5 & 22.7 & 17 & 77.3 & 22 & 14.67 & 3.936 & $0.047 *$ \\
\hline Absent & 11 & 8.6 & 117 & 91.4 & 128 & 85.33 & & \\
\hline
\end{tabular}

\section{Conclusion}

The study results showed that $74 \%$ of the participants had risk factors for delirium, among which $11 \%$ had delirium. In the context of an aging population, use of services by the elderly throughout the health care system continues to grow, particularly among those aged 85 years and above. Delirium has serious consequences and is a "high priority" nursing challenge for all nurses who care for older adults. Though delirium is considered to be a reversible condition, it is also reported to be associated with high mortality. Nurses play a key role in both the prevention and early recognition of this potentially devastating condition. Recognizing the first signs of delirium is important to identify, eliminate or reduce the risk factors such as pain, infection, or other acute illnesses. A significant proportion of delirium cases can be prevented by identifying modifiable risk factors and utilizing a standard nursing practice protocol (delirium identification, risk factor assessment and multicomponent nonpharmacological nursing interventions) promptly.

\section{References}

[1] Ahmed S.,leurent, B.,Sampson,E.L (2014).Risk factors for incident delirium older people in acute hospital medicine units: a systematic review and meta analysis.Age Ageing,May 43(3): 326-22. Retrived on 23.10.2017.

[2] American PsychiatricAssociation . (2000). Diagnostic and statistical manual of mental disorders ( $\left.4^{\text {th }} \mathrm{ed}\right)$ Washington, DC: American Psychiatric Publishing. Retrieved http://psyciatric.org
[3] Eduard E.V., et al.,(2014).Epidemiology and risk factors for delirium across hospital settings. Age ageing. 43(3)326-333. Retrieved on 24.10.2017.

[4] Elie.M., et al (1998). Delirium risk factors in Elderly Hospitalized Patients. J Gen Intern Med, March 13(3)204-212. Retrived on 24.10.2017

[5] Fann,J.R(2000).The epidemiology of delirium. A review of studies and methodological issues. Seminars in Clinical Neuropsychiatry,5,64-74.Evidence Level I:Systematic Review

[6] Inouye,S.K.,Van,C.H.,Alessi,C.A.,et al.(1990).Clarifying confusion: the confusion assessment method. A new method for detection of delirium. Annuals of Internal Method,113,941-948. Retrived on December 7,2016,from www .annals.org

[7] Korevaar JC,et al(2005).Risk factors for delirium in actually admitted elderly patients; a prospective cohort studt.BMC.Geriatric.April13,5:6.Retrived on 24.10.17

[8] Pisani et al.,(2007). Characteristics associated with delirium in older patients in a medical intensive care unit. Archives of Internal Medicine,167(15),1629-1634. Retrieved on November 7,2016,from https://www.ncbi.nlm.nih.gov/pubmed/17698685

[9] Ryan,D..J.,et all (2017).Delirium in an adult acute hospital population: predictors, prevalence and detection.bmjopen.bmj.com/content/3/1/e001772. Retrieved on 25.10.2017.

[10] Varghese,N.C.,Premkumar,B.,Jacob,A.R.,Mathews,P (2012).A study done to determine the prevalence of delirium and the effectiveness of an educational programme on the knowledge and practice of staff nurses regarding delirium identification and management of delirium among elderly at CMC,Vellore 


\section{International Journal of Science and Research (IJSR) \\ ISSN (Online): 2319-7064}

Index Copernicus Value (2016): 79.57 | Impact Factor (2015): 6.391

[11] Yong TY, Huang JE,Lau SY,Li JY ( 2011).Severe hyponatremia and other electrolyte disturbances associated with indapamide.Curr.Drug.Saf,Jul:6 (3), 134-7

[12]Zieschang,T.,et al(2016).The association of hyponatremia, Risk of Confusional State and mortality. A Prospective Controlled Longitudinal Study in Older Patients. Dec; 113(50): 855-862. Retrived on 23.10.2017

Volume 6 Issue 12, December 2017

www.ijsr.net 